\title{
Diagnostic value of serum bile acid composition patterns and serum glycocholic acid levels in cholangiocarcinoma
}

\author{
SIRIPORN PROUNGVITAYA ${ }^{1,2^{*}}$, SUTTHIKAN SOMBATTHEERA ${ }^{1,2^{*}}$, \\ PATCHAREE BOONSIRI $^{3}$, TEMDUANG LIMPAIBOON ${ }^{1,2}$, SOPIT WONGKHAM $^{2}$, \\ CHAISIRI WONGKHAM ${ }^{2}$, ATTAPOL TITAPUN ${ }^{2,4}$ and TANAKORN PROUNGVITAYA ${ }^{1,2}$ \\ ${ }^{1}$ Centre for Research and Development of Medical Diagnostic Laboratories, Faculty of Associated Medical Sciences; \\ ${ }^{2}$ Liver Fluke and Cholangiocarcinoma Research Center; Departments of ${ }^{3}$ Biochemistry and ${ }^{4}$ Surgery, \\ Faculty of Medicine, Khon Kaen University, Khon Kaen 40002, Thailand
}

Received December 1, 2015; Accepted January 26, 2017

DOI: $10.3892 / 01.2017 .6763$

\begin{abstract}
Cholangiocarcinoma (CCA) is a cancer of biliary epithelial cell origin, which is prevalent in northeastern Thailand. The majority of patients with CCA are diagnosed at the advanced-stage of the disease. Although the early detection and diagnosis of CCA is critical to improve the prognosis of patients, there are presently no specific tumor markers for CCA. A previous study demonstrated that the total serum bile acid (TSBA) levels of patients with CCA are significantly increased, compared with those of healthy controls. In addition, although statistically insignificant, the TSBA levels in the sera of patients with CCA tended to be increased, as compared with the sera of patients with benign biliary disease (BBD). In the present study, the high performance liquid chromatographic (HPLC) patterns of bile acid composition were compared in the sera of patients with CCA, patients with BBD and normal controls. The results revealed that serum bile acid patterns in patients with CCA varied, compared with those in patients with BBD and normal controls. As hypothesized, glycocholic acid (GCA) levels in the sera of patients with CCA and BBD were high, compared with those in healthy controls. In addition, GCA levels in the sera of patients with CCA tended to be higher, as compared with patients with BBD; however, this result was not statistically significant. Therefore, determination of the bile acid patterns and GCA levels in sera using HPLC is feasible, and may aid the diagnosis of CCA.
\end{abstract}

Correspondence to: Dr Tanakorn Proungvitaya, Centre for Research and Development of Medical Diagnostic Laboratories, Faculty of Associated Medical Sciences, Khon Kaen University, Khon Kaen 40002, Thailand

E-mail: tanakorn@kku.ac.th

${ }^{*}$ Contributed equally

Key words: serum bile acid, serum glycocholic acid, cholangiocarcinoma

\section{Introduction}

Cholangiocarcinoma (CCA) develops in the biliary epithelial cells and varies in prevalence geographically with the highest incidence rates in southeast Asia (1). The incidence of intrahepatic CCA in the year 2012 was highest in northeastern Thailand, particularly in Khon Kaen (44.3/100,000 males and 17.6/100,000 females) (2) where its occurrence was associated with the high prevalence of carcinogenic liver fluke, Opisthorchis viverrini, infection $(3,4)$.

Primary bile acids, which are synthesized in the liver, are converted into secondary and tertiary bile acids by intestinal bacterial flora. These three types of bile acid have varying functions during carcinogenesis (5). A decrease in the ratio of glycine-conjugated bile acids and taurine-conjugated bile acids has been observed in patients with CCA (6). During the development of CCA, deoxycholic acid (DCA) activates epidermal growth factor receptor, which has been demonstrated to stimulate pro-survival and pro-proliferative signaling pathways, including the phosphoinositide 3-kinase signaling pathway, via complex underlying mechanisms (7). By contrast, tauroursodeoxycholate inhibits human CCA tumor growth via calcium protein kinase $\mathrm{C} \alpha$ and the mitogen activated protein kinase-dependent signaling pathways (8).

Due to the variation in the physicochemical properties of bile acids, including lipophilicity and polarity, the complete and accurate separation and identification of these acids requires the use of advanced chromatographic techniques $(9,10)$. Serum bile acid profiling may provide novel markers and allow early-stage diagnosis of CCA in at risk populations, including those with a high prevalence of Opisthorchis viverrini infection. The present study aimed to compare the bile acid composition patterns between patients with CCA, benign biliary disease (BBD) and normal controls using high performance liquid chromatography (HPLC).

\section{Materials and methods}

Patient selection. Serum samples obtained from 10 patients with CCA, (age, 48-75 years; 6 males and 4 females) and 9 patients with BBD (age, 54-88 years; 8 males and 1 female) 
were selected from registered patients at The Liver Fluke and Cholangiocarcinoma Research Center, Srinagarind Hospital, Faculty of Medicine, Khon Kaen University (Khon Kaen, Thailand). The sample was collected from the patient prior to surgery. Sera were also obtained from 8 healthy controls, who received health check-ups at the office of The Medical Technology and Physical Therapy Health Service, Faculty of Associated Medical Sciences, Khon Kaen University. All serum samples were collected from January 2006 to March 2013 for the retrospective study.

The present study was approved by the Ethics Committee for Human Research (approval no. HE561280), Khon Kaen University (Khon Kaen, Thailand).

Total serum bile acid (TSBA) evaluation. TSBA levels in $3 \mu 1$ serum samples were determined using the Total Bile Acids assay kit (Diazyme Laboratories, Poway, CA, USA), following the enzyme cycling method as described in the manufacturer's protocol. This analysis was performed using the Beckman Synchron CX4 Clinical Chemistry Analyzer (Beckman Coulter, Inc., Brea, CA, USA) and the results were presented as $\mu \mathrm{mol} / 1$.

Bile acid standards. Bile acid standards were as follows: Primary bile acid [cholic acid; chenodeoxycholic acid (CDCA)]; secondary bile acid [DCA; lithocholic acid (LCA)]; tertiary bile acid [ursodeoxycholic acid (UDCA)]. In addition, conjugated bile acid standards with taurine [taurocholic acid (TCA); taurodeoxycholic acid] or glycine [glycocholic acid (GCA); glycodeoxycholic acid] were used. All bile acid standards gallic acid were purchased from Sigma-Aldrich (Merck KGaA, Darmstadt, Germany). Gallic acid was used as the internal standard for HPLC analysis of all bile acids in the serum samples, with the exception of the standard compounds.

Solid phase extraction of bile acids. Bile acids were extracted using the solid phase extraction method previously described by Humbert et al (11) and Steiner et al (12) with minor modifications. A total of $100 \mu 1$ of each serum sample was mixed with $100 \mu 10.4 \mathrm{M}$ ammonium carbonate buffer ( $\mathrm{pH}$ 9.3) and $700 \mu \mathrm{l}$ deionized water. The serum samples were incubated at $60^{\circ} \mathrm{C}$ for $30 \mathrm{~min}$ and centrifuged at 4,000 x g for $15 \mathrm{~min}$ at room temperature. For the solid phase extraction, $50 \mathrm{mg} \mathrm{Sep-Pak}$ C18 Vac cartridges (Waters Corporation, Milford, MA, USA) were activated by pre-washing with $2 \mathrm{ml} 100 \%$ methanol, $2 \mathrm{ml}$ deionized water and $2 \mathrm{ml} 0.1 \mathrm{M}$ ammonium carbonate buffer ( $\mathrm{pH}$ 9.3). Subsequently, the supernatant from the centrifuged serum tube was loaded onto the cartridge. The cartridge was washed once with $2 \mathrm{ml}$ deionized water, and the bound bile acids were eluted in $3 \mathrm{ml}$ methanol. The methanol fraction was collected and dried under a nitrogen stream at $50^{\circ} \mathrm{C}$.

HPLC analysis. Gallic acid solution was prepared by mixing equal volumes of $1.25 \mathrm{mM}$ gallic acid in methanol and $0.03 \mathrm{M}$ ammonium acetate buffer (pH 3.5). A $100 \mu \mathrm{l}$ gallic acid solution was used to dissolve the dried serum samples. The solutions were subsequently mixed and centrifuged at $13,400 \mathrm{x} \mathrm{g}$ for $10 \mathrm{~min}$ at $10^{\circ} \mathrm{C}$. The supernatant was collected and passed through nylon syringe filters (pore size, $0.22 \mu \mathrm{m}$; diameter, $13 \mathrm{~mm}$; Bonna-Agela
Table I. Retention times of bile acid standards as detected by high-performance liquid chromatography.

\begin{tabular}{llc}
\hline No. & Bile acid standard & Retention time, min \\
\hline S1 & Gallic acid & 5.8 \\
S2 & TCA & 12.6 \\
S3 & TDCA & 16.8 \\
S4 & GCA & 19.5 \\
S5 & GDCA & 24.4 \\
S6 & UDCA & 24.9 \\
S7 & CA & 26.3 \\
S8 & CDCA/DCA & 31.1 \\
S9 & LCA & 35.7 \\
\hline
\end{tabular}

S, standard; TCA, taurocholic acid; TDCA, taurodeoxycholic acid; GCA, glycocholic acid; GDCA, glycodeoxycholic acid; UDCA, ursodeoxycholic acid; CA, cholic acid; CDCA, chenodeoxycholic acid; DCA, deoxycholic acid; LCA, lithocholic acid.

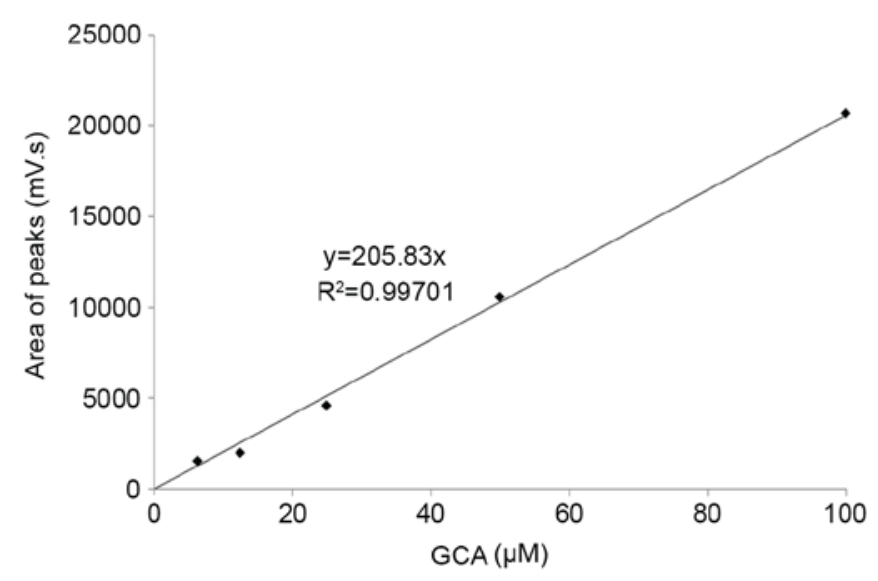

Figure 1. Standard curve for GCA. GCA, glycocholic acid.

Technologies, Inc., Wilmington, DE, USA). A total of $20 \mu 1$ of each filtered serum sample was injected into the HPLC system (reverse phase chromatography), which consisted of a Spherisorb ${ }^{\circledR}$ ODS2 $80 \AA$ column $(5 \mu \mathrm{m} ; 4.6 \times 250 \mathrm{~mm}$; Waters Corporation), an HPLC pump (model 515; Waters Corporation) and an evaporative light scattering detector (model 2424; Waters Corporation). Chromatographic separation was performed with gradient elution at a flow rate of $1.0 \mathrm{ml} / \mathrm{min}$ at room temperature. The first mobile phase was absolute methanol and the second was $0.03 \mathrm{M}$ ammonium acetate buffer ( $\mathrm{pH} 3.5)$. The mobile phase gradient elutions were programmed at the following time points: $0,35,45,45.5$ and $55 \mathrm{~min}$. The percentages of mobile phase 1 and phase 2 were $60: 40,100: 0,100: 0,60: 40$ and $60: 40 \%$ at each time point, respectively. For all the experiments, evaporative light scattering detector parameters were set as follows: Gain at 2, gas pressure at $25 \mathrm{psi}$, nebulizer temperature at $30^{\circ} \mathrm{C}$ and drift tube temperature at $80^{\circ} \mathrm{C}$.

HPLC interpretation. To elucidate the bile acid composition patterns in each group, HPLC chromatograms were interpreted 


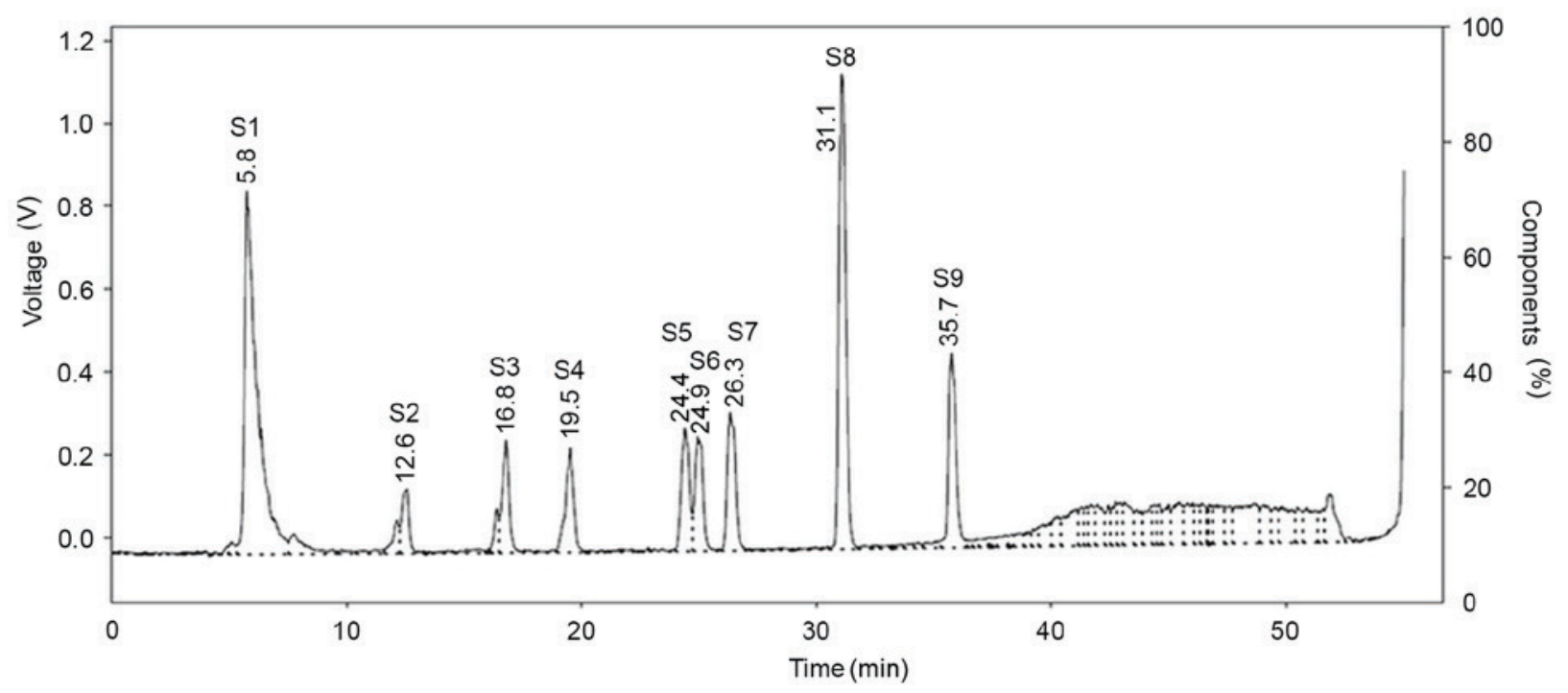

Figure 2. High-performance liquid chromatography profile of bile acid standards. S1, gallic acid (internal standard); S2, taurocholic acid; S3, taurodeoxycholic acid; S4, glycocholic acid; S5, glycodeoxycholic acid; S6, ursodeoxycholic acid; S7, cholic acid; S8, chenodeoxycholic acid/deoxycholic acid; S9, lithocholic acid. The number above each peak represents the retention time ( $\mathrm{min}$ ) of each standard.

using two steps. The retention time of each peak, obtained from the serum samples, was compared with the synthetic bile acid standards. The peaks with a retention time equal to the standards were considered to be the corresponding bile acid compound. Subsequently, the retention times of peaks that were not associated with the standards, were calculated as the percentage of relative migration by comparison with the longest retention time of the standards.

The bile acid composition patterns were generated using the following criteria: Any peak with a retention time equal to the corresponding bile acid standard, or any relative retention time that identified at $\geq 5 / 10(50 \%)$ cases in the CCA group, $5 / 9(55 \%)$ cases in the BBD group and at $\geq 4 / 8(50 \%)$ cases in the normal group, was plotted on the time line axis. Each time point was assigned a cardinal number to allow comparison of the bile acid composition pattern of individual patients in each group. The presence or absence of the assigned number among CCA, BBD and normal control groups was further analyzed to produce a model flow chart to aid differential diagnosis between the three groups.

GCA standard curve. The GCA standard curve was prepared by plotting the coordinates of the concentrations of GCA on the $\mathrm{x}$-axis, and the areas under the curve obtained from HPLC peaks on the y-axis (Fig. 1). The standard curve was produced from the linear regression equation $y=205.83 x$, and the correlation coefficient $\left(\mathrm{r}^{2}\right)$ was 0.997 by using Microsoft Excel 2010 software (Microsoft Corporation, Redmond, WA, USA). Concentrations of GCA in the serum samples were calculated using the standard curve. The concentrations of TSBA and GCA, and the ratio of GCA to TSBA, were subsequently compared between the CCA and BBD groups. The GraphPad Prism software (version 5.0; GraphPad Software Inc., CA, USA) was used for statistical analyses. The different values between the two independent sample groups were analyzed using Mann-Whitney $\mathrm{U}$ test. $\mathrm{P}<0.05$ was considered to indicate a statistically significant difference.

\section{Results}

HPLC chromatogram of bile acid standards. The HPLC chromatograms of nine bile acid standards and gallic acid as an internal control are presented in Fig. 2, with retention times summarized in Table I. Although 10 standard bile acids were analyzed using HPLC, only 9 overall peaks were observed on the chromatogram, as CDCA and DCA were not able to be separated and were co-eluted due to having the same retention time (31.1 $\mathrm{min})$.

HPLC chromatogram of serum samples. The representative HPLC profiles of bile acids in the sera of patients with CCA and $\mathrm{BBD}$, as well as normal control groups, are presented in Fig. 3. A total of 34 peaks were identified, including 9 peaks corresponding to the standard bile acids, in the HPLC profiles of all the serum samples. These were assigned peak numbers 1-34. Subsequently, the bile acid composition patterns of the CCA, BBD and normal control groups were examined. As presented in Fig. 4, GCA and three unknown peaks (nos. 7, 13 and 34) were identified in the CCA group. In addition, GCA, UDCA and one unknown peak (no. 34) were identified in the BBD group. Notably, GCA and unknown peak no. 34 were found in the CCA and BBD groups but not in the control group. Two unknown peaks (nos. 31 and 32) were unique to the control group. As the GCA peak was distinct in the CCA and BBD patient groups, but not in the control group, GCA was selected to distinguish between the disease groups and the normal group at the first stage. A flow chart (Fig. 5) was produced using these results in order to provide a diagnostic guideline. Based on this, the diagnostic values of the bile acid pattern analysis were 70,78 and $100 \%$ for the CCA, BBD and normal patient groups, respectively.

GCA quantification. As aforementioned, the GCA peak was observed in the CCA and BBD groups, but not in normal controls. Therefore, the GCA peak was selected to quantify 
A CCA

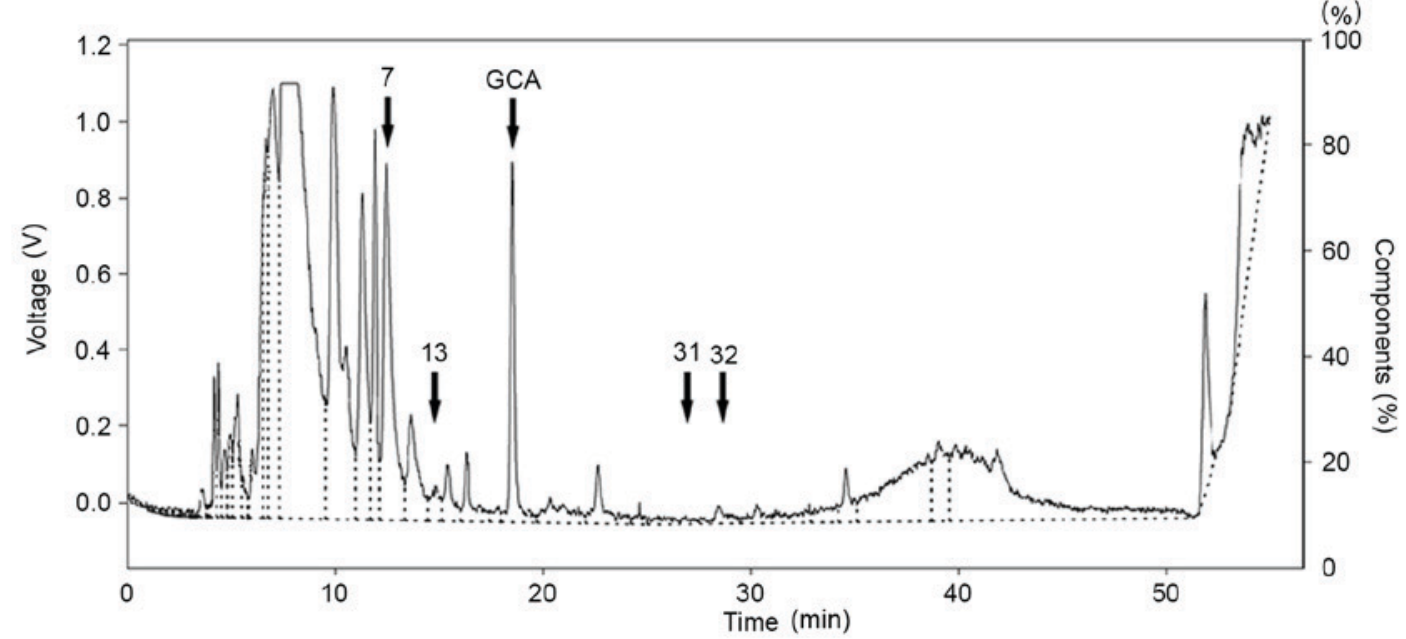

B BBD

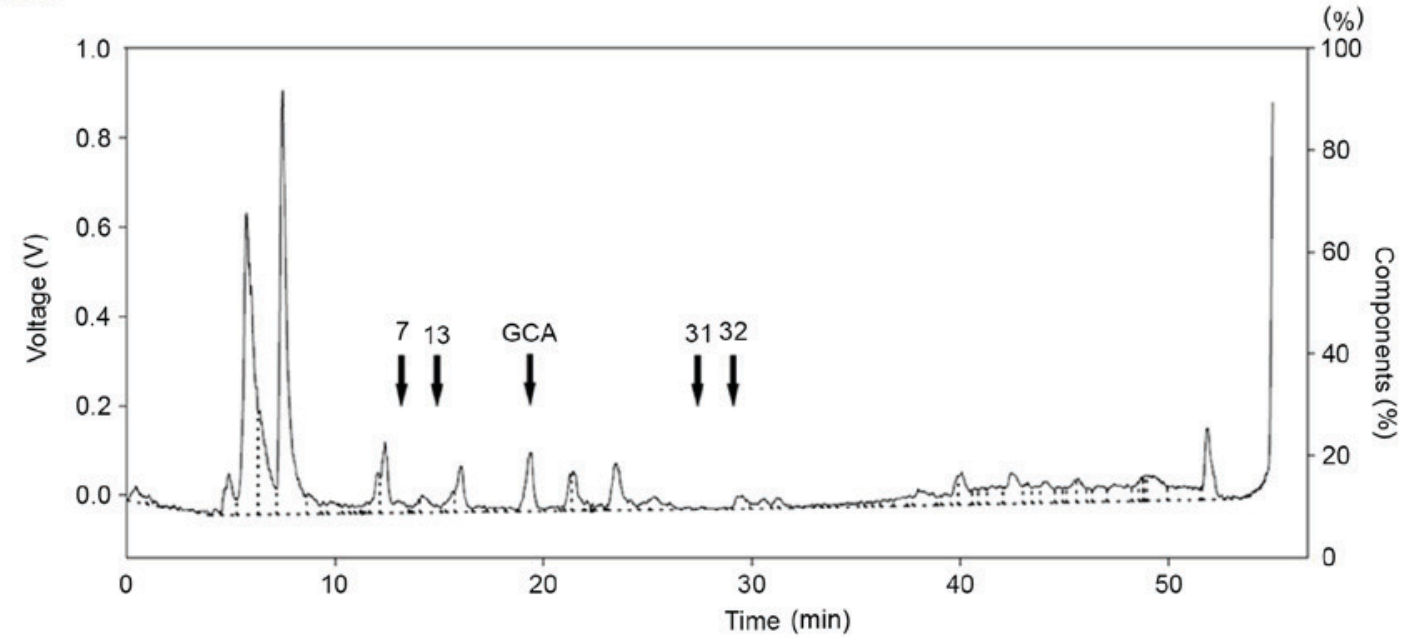

\section{Normal control}

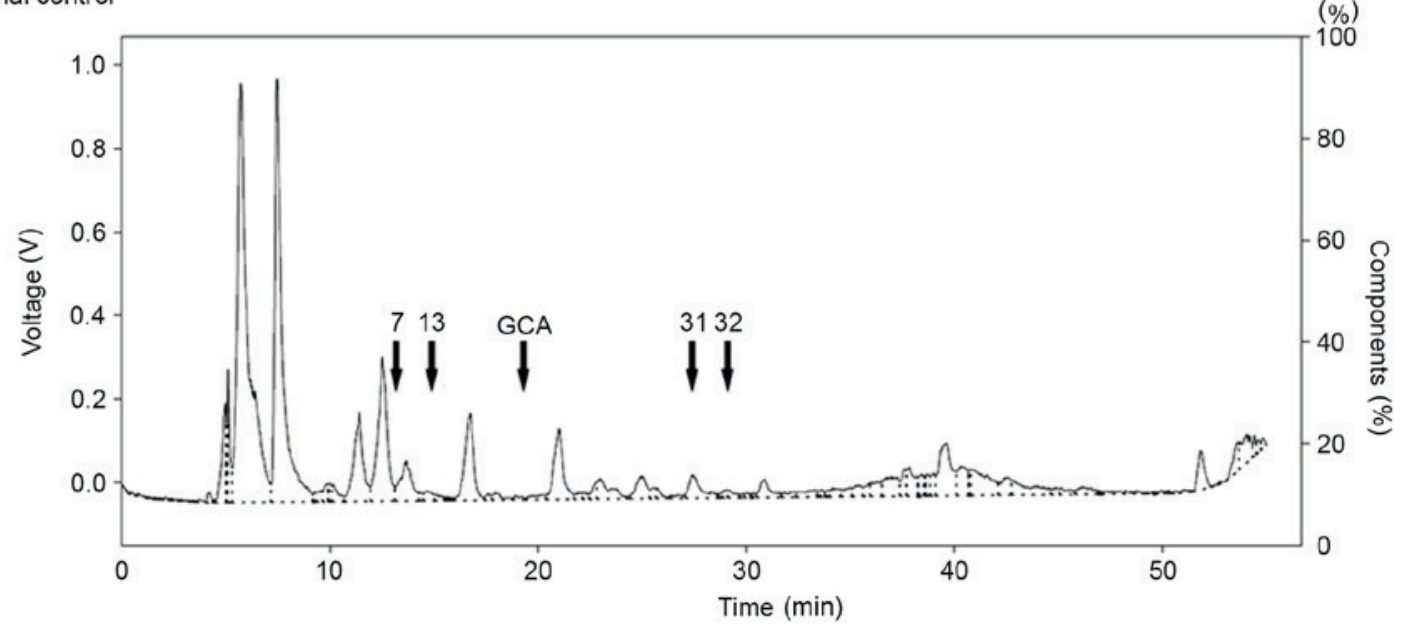

Figure 3. Representative HPLC profiles of bile acids in the serum of (A) a patient with CCA patient (B) a patient with BBD and (C) a healthy patient. GCA, glycocholic acid; CCA, cholangiocarcinoma; BBD, benign biliary disease; HPLC, high performance liquid chromatography.

the serum GCA levels using the standard curve (Fig. 1). A comparison of the levels of TSBA and GCA, and of the GCA:TSBA ratio, between the CCA and BBD groups is presented in Fig. 6. TSBA and GCA levels in the CCA group were revealed to be higher than those observed in the BBD group (Fig. 6A and B). The ratio of GCA:TSBA presented in Fig. $6 \mathrm{C}$ is also higher in the CCA group, as compared with the BBD group. 


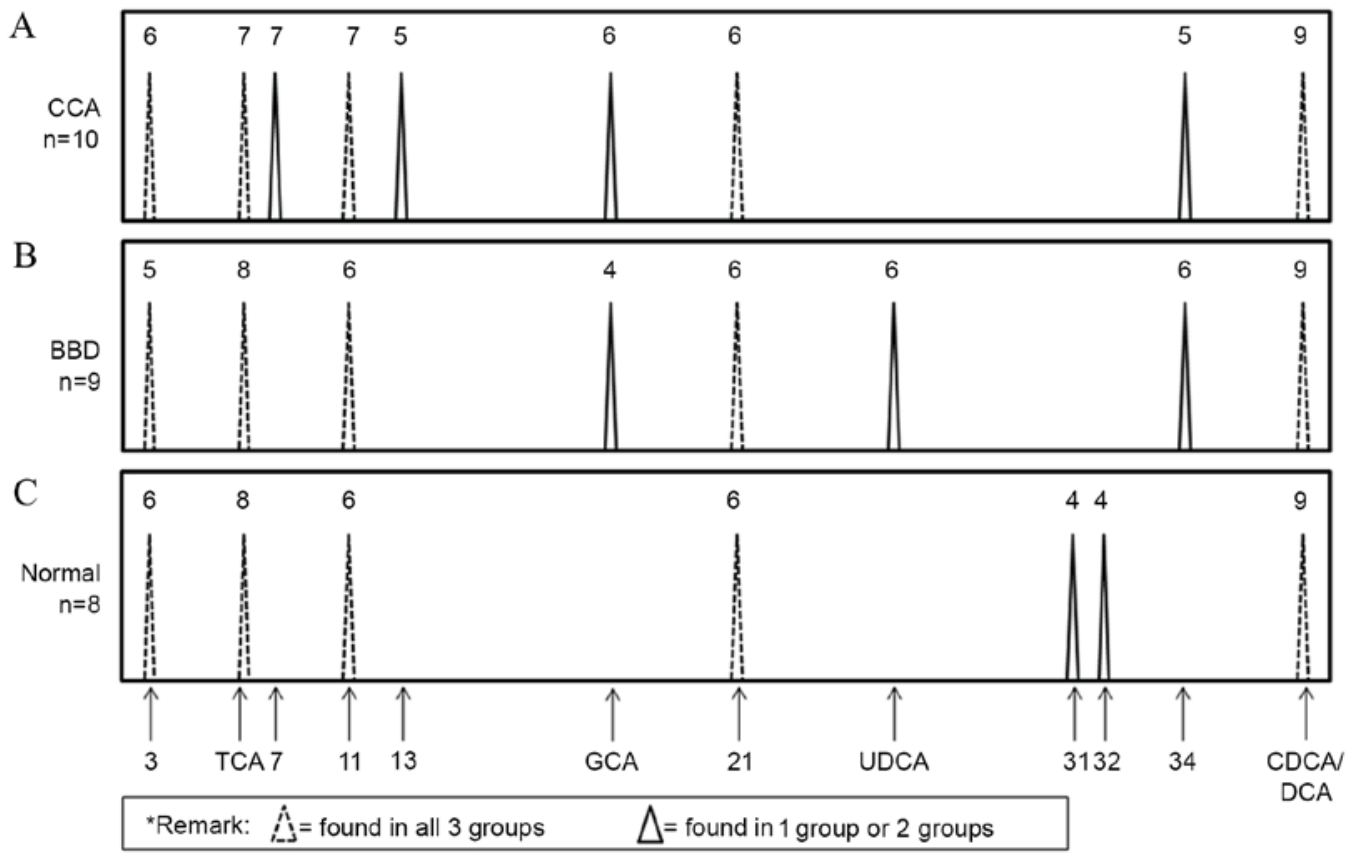

Figure 4. Model patterns of bile acid composition in the sera of patients with (A) CCA and (B) BBD, as well as (C) normal controls. The number above each peak represents the frequency of peak-positive cases. CCA, cholangiocarcinoma; BBD, benign biliary disease; TCA, taurocholic acid; GCA, glycocholic acid; UDCA, ursodeoxycholic acid; CDCA, chenodeoxycholic acid; DCA, deoxycholic acid.

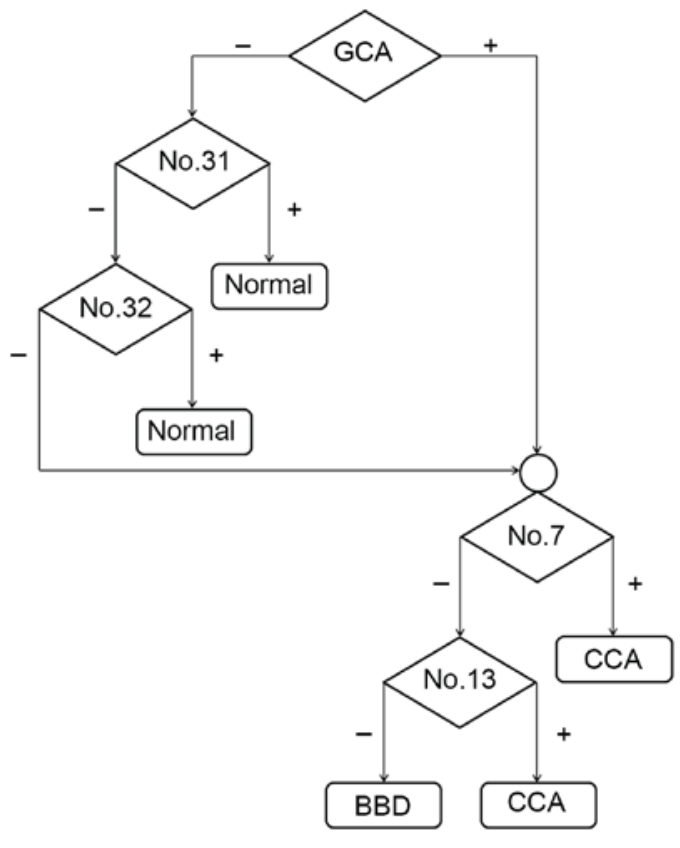

Figure 5. Flow chart of bile acid peaks to allow differentiation between CCA, $\mathrm{BBD}$ and normal groups. CCA, cholangiocarcinoma; BBD, benign biliary disease; GCA, glycocholic acid.

\section{Discussion}

Through the use of HPLC separation, GCA was detected in the sera of patients with CCA and BBD, but not in the control group. Furthermore, while statistically significant differences were not observed $(\mathrm{P}=0.171)$, the GCA levels in the CCA group tended to be higher, compared with those of the BBD group. These results were concordant with Changbumrung et al (6),

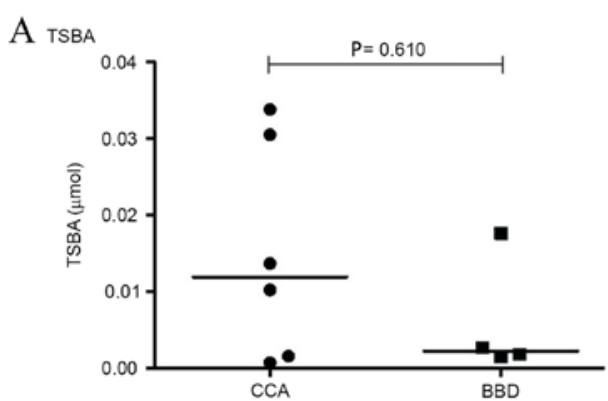

B GCA
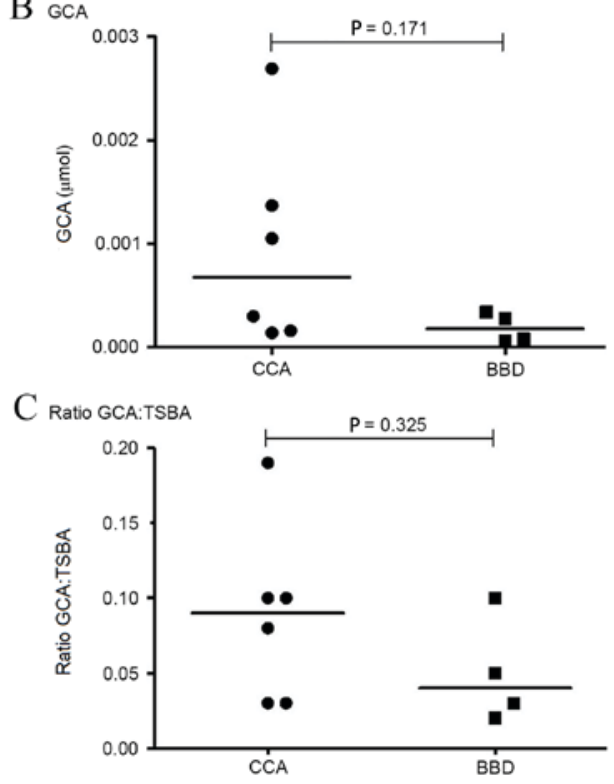

Figure 6. (A) Concentration of TSBA and (B) GCA in the sera of patients with CCA and BBD. (C) The GCA:TSBA ratio in the CCA and BBD groups. TSBA, total serum bile acid; GCA, glycocholic acid; BBD, benign biliary disease; CCA, cholangiocarcinoma. 
in that TSBA and the levels of conjugated bile acids, including GCA, glycochenodeoxycholic acid (GCDCA), TCA and taurochenodeoxycholic acid (TCDCA) in the sera of patients with CCA and hepatocellular carcinoma (HCC) were higher, compared with those observed in healthy controls. In addition to $\mathrm{CCA}$ and $\mathrm{HCC}, \mathrm{GCA}$ and other bile acids exhibit elevated levels in the sera of patients with various hepatobiliary diseases (hepatitis virus infection, alcoholic liver disease, primary biliary cirrhosis, biliary tract stones and primary sclerosing cholangitis), and demonstrate variable bile acid composition patterns $(12,13)$. Patients receiving UDCA as a treatment for biliary tract diseases exhibit high levels of GCA, TCA, GCDCA and TCDCA levels (13). Furthermore, high levels of GCA, TCA and GCDCA have been observed in patients with alcoholic liver disease, whereas patients with non-alcoholic fatty liver disease exhibit high levels of CA, GCA and GCDCA (13). In addition, a previous study demonstrated that GCA expression is upregulated in the urine samples of patients with liver cancer (14).

HPLC is a convenient and reliable technique for routine bile acid composition analysis (15). Serum bile acid profiles of healthy subjects and patients with various hepatobiliary diseases exhibit variation in the composition of conjugated bile acids (15). In the current study, serum bile acid profiles varied between patients with CCA, BBD and healthy subjects, with unknown peaks (nos. 7 and 13) observed in the sera of patients with CCA but not BBD. As the unique peaks identified in sera from patients with CCA did not correspond to the bile acid standards, their chemical composition requires further elucidation. In addition, the UDCA peak was only observed in the sera of patients with BBD. This may be due to the therapeutic agents used for BBD, as UDCA is frequently used to treat chronic cholestatic diseases $(16,17)$.

In conclusion, determination of the bile acid composition patterns in patient serum samples using HPLC may aid the diagnosis of CCA, and GCA may provide a novel marker for this disease. Further metabolomics and investigation of the mechanisms underlying the involvement of GCA in the pathophysiology of CCA and carcinogenesis of the bile duct are required. In order to provide a comprehensive evaluation of the diagnostic potential of bile acid composition profiles and GCA levels, large-scale studies are required.

\section{Acknowledgements}

The authors of the present study would like to thank The Centre for Research and Development of Medical Diagnostic Laboratories, Faculty of Associated Medical Sciences, Khon Kaen University (Khon Kaen, Thailand) for providing technical support with the instruments used in the present study. The authors would also like to thank The Liver Flukes and Cholangiocarcinoma Research Center, Faculty of Medicine, Khon Kaen University for providing sera samples from patients with CCA. In addition, the authors would like to thank Miss Roongpet Tangrassameeprasert (Department of Biochemistry, Faculty of Medicine, Khon Kaen University) for technical support with the HPLC analyses and Professor Yukifumi Nawa Academic Affairs, Faculty of Medicine, Khon Kaen University for manuscript editing. The present study was supported by the Publication Clinic of Research Affairs, Khon Kaen University.

\section{References}

1. Blechacz BR and Gores GJ: Cholangiocarcinoma. Clin Liver Dis 12: 131-150, ix, 2008.

2. Wiangnon S, Suwanrungruang $K$ and Kamsa-Ard S: Cholangiocarcinoma in Khon Kaen Province. Srinagarind Med J 27 suppl (Cholangiocarcinoma): S326-S330, 2012

3. Sripa B, Bethony JM, Sithithaworn P, Kaewkes S, Mairiang E, Loukas A, Mulvenna J, Laha T, Hotez PJ and Brindley PJ: Opisthorchiasis and Opisthorchis-associated cholangiocarcinoma in Thailand and Laos. Acta Trop 120 (Suppl 1): S158-S168, 2011.

4. Sripa B, Kaewkes S, Sithithaworn P, Mairiang E, Laha T, Smout M, Pairojkul C, Bhudhisawasdi V, Tesana S, Thinkamrop B, et al: Liver fluke induces cholangiocarcinoma. PLoS Med 4: e201, 2007.

5. Debruyne PR, Bruyneel EA, Li X, Zimber A, Gespach C and Mareel MM: The role of bile acids in carcinogenesis. Mutat Res 480-481: 359-369, 2001.

6. Changbumrung S, Tungtrongchitr R, Migasena $\mathrm{P}$ and Chamroenngan S: Serum unconjugated primary and secondary bile acids in patients with cholangiocarcinoma and hepatocellular carcinoma. J Med Assoc Thai 73: 81-90, 1990.

7. Werneburg NW, Yoon JH, Higuchi H and Gores GJ: Bile acids activate EGF receptor via a TGF-alpha-dependent mechanism in human cholangiocyte cell lines. Am J Physiol Gastrointest Liver Physiol 285: G31-G36, 2003.

8. Alpini G, Kanno N, Phinizy JL, Glaser S, Francis H, Taffetani S and LeSage G: Tauroursodeoxycholate inhibits human cholangiocarcinoma growth via Ca2+-, PKC-, and MAPK-dependent pathways. Am J Physiol Gastrointest Liver Physiol 286: G973-G982, 2004.

9. Scalia S and Games DE: Determination of free bile acids in pharmaceutical preparations by packed column supercritical fluid chromatography. J Pharm Sci 82:44-47, 1993.

10. Roda A, Piazza F and Baraldini M: Separation techniques for bile salts analysis. J Chromatogr B Biomed Sci Appl 717: 263-278, 1998.

11. Humbert L, Maubert MA, Wolf C, Duboc H, Mahé M, Farabos D, Seksik P, Mallet JM, Trugnan G, Masliah J and Rainteau D: Bile acid profiling in human biological samples: Comparison of extraction procedures and application to normal and cholestatic patients. J Chromatogr B Analyt Technol Biomed Life Sci 899: 135-145, 2012.

12. Steiner C, von Eckardstein A and Rentsch KM: Quantification of the 15 major human bile acids and their precursor $7 \alpha$-hydroxy-4-cholesten-3-one in serum by liquid chromatography-tandem mass spectrometry. J Chromatogr B Analyt Technol Biomed Life Sci 878: 2870-2880, 2010.

13. Sugita T, Amano K, Nakano M, Masubuchi N, Sugihara M and Matsuura T: Analysis of the serum bile acid composition for differential diagnosis in patients with liver disease. Gastroenterol Res Pract 2015: 717431, 2015.

14. Zhang A, Sun H, Yan G, Han Y, Ye Y and Wang X: Urinary metabolic profiling identifies a key role for glycocholic acid in human liver cancer by ultra-performance liquid-chromatography coupled with high-definition mass spectrometry. Clin Chim Acta 418: 86-90, 2013

15. Lee BL, New AL and Ong CN: Comparative analysis of conjugated bile acids in human serum using high-performance liquid chromatography and capillary electrophoresis. J Chromatogr B Biomed Sci Appl 704: 35-42, 1997.

16. Olsson R: Ursodeoxycholic acid in the treatment of chronic cholestatic liver disease. Documented delay in disease progress inspires hope. Lakartidningen 99: 1325-1330, 2002 (In Swedish).

17. Poupon R, Chazouillères O and Poupon RE: Chronic cholestatic diseases. J Hepatol 32 (1 Suppl): S129-S140, 2000. 